> Malgré les avancées de la recherche, un grand nombre de patients atteints de troubles du spectre autistique (TSA) n'ont pas accès aux explorations aujourd'hui disponibles, du fait d'idées reçues, de l'insuffisance des structures à même de les explorer et de l'inadaptation des consultations hospitalières à leurs troubles $d u$ comportement. Pour améliorer l'accès aux soins et au progrès des connaissances, nous avons inversé le paradigme et offrons depuis 20 ans des consultations de génétique clinique sur site dans les hôpitaux de jour et les institutions spécialisées de la région parisienne. Depuis 1998, une équipe mobile de génétique médicale propose aux patients et à leurs familles des consultations dans leur environnement habituel. L'unité mobile opère sous l'égide de l'hôpital universitaire Necker Enfants-Malades, qui leur donne accès aux services de biochimie, de cytogénétique moléculaire et de séquençage de nouvelle génération (NGS). En vingt ans, 502 patients appartenant à 26 institutions ont bénéficié de consultations sur site et d'un accès aux plateformes de génétique moléculaire. Moins de $1 \%$ des parents ont décliné la proposition. Des affections génétiques ont été identifiées chez 71 patients présentant un TSA : anomalies cytogénétiques causales (34/388: 8,8\%; de novo : 19, héritées : 4), X Fragile (4/312:1,3\%) et mutations monogéniques reconnues responsables de TSA (33/141; $23,4 \%$ : de novo : 23 ; héritées: 10 , dont 5 liées à I'X et 5 récessives autosomiques). L'IRM cérébrale a été possible chez 347 patients et considérée comme anormale chez $42 \%$ d'entre eux (146/347). Tous les patients diagnostiqués présentaient un TSA atypique ou syndromique, avec déficience intellectuelle modérée à sévère. Grâce à ce mode d'intervention, un grand nombre de consultations manquantes ont été rattrapées et les familles

\section{Vingt ans \\ de consultations \\ de génétique \\ clinique sur site \\ dans les hôpitaux \\ de jour pour \\ les personnes \\ atteintes \\ de troubles \\ du spectre \\ autistique de la région parisienne}

Arnold Munnich, Caroline Demily, Lisa Frugère, Charlyne Duwime, Valérie Malan, Giulia Barcia, Céline Vidal, Émeline Throo, Claude Besmond, Laurence Hubert, Gilles Roland-Manuel, Jean-Pierre Malen, Mélanie Ferreri, Sylvain Hanein, Nathalie Boddaert, Moise Assouline

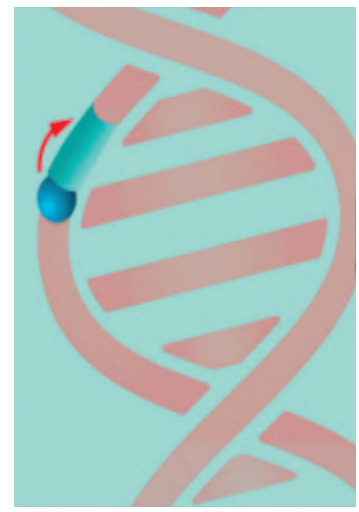

Fédération de Génétique Médicale et Institute Imagine, UMR Inserm 1163, Université Paris-Descartes, Hôpital Necker Enfants-Malades et Fondation Elan Retrouvé, 149 rue de Sèvres, 75015 Paris, France.

arnold.munnich@inserm.fr

ont pu bénéficier d'une consultation de génétique. Eu égard aux contraintes imposées par les troubles du comportement dans les TSA, les consultations sur site constituent, pour les patients et leurs apparentés, un moyen d'améliorer l'accès aux soins et de réduire le risque de méconnaissance d'une pathologie organique à présentation psychiatrique. <

Vignette (Photo @ Inserm-Frédérique Koulikoff).

La présente étude a fait l'objet d'une publication récente dans Molecular Autism [16]. 
Les troubles du spectre autistique (TSA) constituent un problème majeur de santé qui touche un enfant sur 100 à 200 (avec un sex-ratio de 4 garçons pour 1 fille). Des progrès importants dans la génétique des syndromes autistiques ont été accomplis dans les dernières années $[1,2]$. Pourtant, la méconnaissance de ces progrès récents, les idées préconçues sur la pathogénie de l'autisme et l'absence de marqueurs biologiques de dépistage dissuadent nombre de professionnels d'explorer ces enfants et les conduisent même à contester la possible origine organique de la maladie. D'autres raisons expliquent pourquoi tant d'enfants et adolescents souffrant de TSA ne bénéficient pas d'explorations systématiques, notamment a) le manque de neuropédiatres et de généticiens cliniciens, $b$ ) la congestion et l'insuffisance des consultations pédiatriques hospitalières spécialisées et c) la rareté des plateformes d'explorations performantes. Pour offrir aux patients un meilleur accès aux soins et mieux diffuser le progrès des connaissances scientifiques, nous avons inversé le paradigme et offrons des consultations de génétique sur site dans des hôpitaux de jour et des institutions spécialisées de la région parisienne.

$\varepsilon n$ vingt ans, 502 patients ont ainsi bénéficié de consultations sur site et d'un accès à une plateforme de génétique moléculaire hospitalière. Grâce à ce mode d'intervention, un grand nombre de consultations manquantes ont été rattrapées et les familles ont pu bénéficier d'une consultation de génétique. Eu égard aux contraintes imposées par les troubles du comportement dans les TSA, les consultations sur site constituent, pour les patients et leurs apparentés, un mode d'accès aux soins et un moyen de réduire le risque de méconnaissance d'une pathologie organique à présentation psychiatrique.

\section{L'équipe mobile de génétique médicale}

Depuis 1998, l'équipe mobile de génétique médicale qui se déplace sur site, est basée dans le service de génétique médicale de l'hôpital Necker-Enfants Malades et opère dans le cadre d'un partenariat régional avec l'Institut des maladies génétiques-Imagine et la Fondation Elan Retrouvé. La mise en œuvre du programme repose sur deux coordinatrices, une neuropsychologue, une conseillère en génétique et un généticien clinicien. Les patients et leurs parents qui le souhaitent sont reçus dans leur environnement familier, réduisant ainsi l'angoisse de l'attente dans un lieu qui leur est inhabituel. L'exploration systématique a permis de porter le diagnostic d'une pathologie organique à présentation psychiatrique dans un tiers des cas, principalement dans des TSA syndromiques, atypiques, avec une déficience intellectuelle modérée à sévère.

Au total, 26 hôpitaux de jour et établissements médicaux ou médicosociaux spécialisés ont été inclus à leur demande. Tous ont un statut d'association et ont été créés dans les années 1960 à l'initiative de parents soucieux d'éviter à leur enfant un placement en hôpital psychiatrique. Tous les patients répondaient aux critères de TSA fondés sur le DSM-5 (cinquième édition du Manuel diagnostique et statistique des troubles mentaux) [3] et présentaient des niveaux cognitifs variables, du déficit léger aux déficiences intellectuelles graves ou profondes. L'évaluation clinique standardisée a pris en compte les symptômes multi-dimensionnels (CARS: childhood autism rating scale, ADOS: autism diagnostic observation schedule et/ou ADIR : autism diagnostic interview-revised).

Le consentement éclairé des parents a été obtenu préalablement à la consultation. Dans le but d'éviter les malentendus, une présentation de la démarche a été proposée sur site avant la consultation avec le généticien clinicien. Les consultations ont eu lieu en présence de l'enfant, de ses parents, du pédopsychiatre et de l'équipe paramédicale et éducative de l'établissement. Les pédopsychiatres ont étroitement coopéré avec le généticien, agissant en qualité d'expert et se gardant de s'immiscer dans la gestion du dossier (prescription médicamenteuse, orthophonie et psychothérapie). Par souci de confidentialité, une consultation avec le généticien seul a été systématiquement proposée. Elle a été cependant rarement souhaitée. Une consultation standard passe en revue les antécédents personnels et familiaux de l'enfant, l'arbre généalogique de la famille, l'album de photos et comprend un examen clinique complet, en présence d'un membre de l'équipe locale. Une attention particulière est portée aux principaux signes cliniques de TSA syndromique. La consultation tente de répondre aux questions suivantes: a) le TSA est-il isolé ou fait-il partie d'un syndrome connu? b) le cas est-il sporadique ou familial ? c) avec ou sans déficience intellectuelle ? et $d$ ) existe-t-il des facteurs de risque (âge paternel avancé, fécondation in vitro, prématurité, consommation de stupéfiants ou ingestion de médicaments pendant la grossesse) ? Le bilan systématique effectué en ambulatoire comporte la recherche d'expansion du gène FMRI (fragile $X$ mental retardation 1), l'analyse chromosomique comparative sur puces à ADN (ACPA, encore appelées CGH array) [4], le bilan métabolique (chromatographie des acides aminés et organiques, taux de succinylpurine, de sialotransferrine, des intermédiaires de synthèse de la créatine). Lorsqu'elle est négative, et avant l'analyse moléculaire par séquençage (NGS), cette première série de tests est suivie d'une IRM (imagerie par résonance magnétique) cérébrale avec spectroscopie par résonance magnétique nucléaire (RMN) et tomodensitométrie après une brève sédation, ainsi que d'un électro-encéphalogramme.

Pour l'analyse NGS, sont testés les gènes connus de TSA, isolés ou syndromiques, rapportés dans deux familles non apparentées au moins. Ils sont séquencés sur un panel de 445 gènes [5-9]. Pour éviter les découvertes fortuites ou les soucis d'interprétation de variants de signification inconnue (VOUS), seuls les gènes de TSA publiés dont la pénétrance est complète sont testés [5-9]. Pour faciliter l'interprétation, le trio parents/enfant est analysé simultanément. 
Certains patients ont bénéficié d'un séquençage de l'exome entier réalisé dans le cadre de projets de recherche. À ce stade, ni le séquençage complet du génome, ni l'analyse de polymorphismes (single nucleotide polymorphisms, SNP) prétendument associés aux TSA dans les grandes études d'association (genome-wide association studies, GWAS) n'ont été réalisés. Les résultats et conclusions ont été communiqués aux patients et aux familles dans les six mois qui ont suivi l'examen, dans la même configuration que la consultation multidisciplinaire initiale.

\section{Observations et résultats : un bilan}

Les consultations sur site étaient facultatives mais ont été accueillies favorablement par la majorité des parents. Moins de $1 \%$ des couples ont décliné l'offre, arguant du fait "qu'aucun bénéfice immédiat ne s'en suivrait ». Au total, 502 enfants, admis dans 26 hôpitaux de jour et institutions spécialisées de la région parisienne, ont ainsi été inclus dans le programme. La possibilité d'initier ou d'actualiser les explorations génétiques répondait largement à leurs attentes, beaucoup des questions concernant la possible origine organique des troubles étant demeurées sans réponse.

Seule une petite fraction des patients avait été vue en consultation par un neuropédiatre ou un généticien clinicien avant la visite sur site et, du fait des avancées récentes, moins de $5 \%$ des dossiers médicaux étaient à jour. Dans $95 \%$ des cas, les examens de laboratoire étaient absents, incomplets ou obsolètes, sans consultations hospitalières de suivi, et dans $30 \%$ des cas, les dossiers médicaux étaient totalement vides. Un grand nombre de parents ont déclaré que leur enfant n'avait jamais été examiné déshabillé...

Le simple établissement d'un arbre généalogique a parfois suffi à reconnaître des formes récessives ou liées au sexe de TSA. Des informations, jamais divulguées auparavant et présentant pourtant un intérêt médical réel, ont également été révélées pour la première fois à l'occasion des consultations (antécédents familiaux, événements médicaux graves durant la grossesse, fécondation in vitro). Il est cependant difficile de dire si ces questions n'avaient effectivement jamais été posées, ou si les réponses des parents avaient été filtrées selon des attentes supposées du pédopsychiatre. En raison du manque de spécificité clinique des TSA d'origine organique, la consultation de génétique s'est rarement terminée par une conviction clinique. Pour cette raison, les examens de laboratoire ultérieurs ont été systématiques.

Pour près de $60 \%$ des patients, le résultat de la recherche du syndrome de I'X fragile n'était pas disponible (312/502). Il s'est révélé positif dans 1,3\% des cas (4/312). L'ACPA (CGH array) a permis de détecter des variations du nombre de copies (CNV) pathogènes dans 8,8 \% (34/388; de novo : 19, héritées : 4) (Tableau I). La plupart des patients diagnostiqués à ce stade présentaient un TSA atypique et/ou syndromique avec déficience intellectuelle modérée à sévère. Le bilan métabolique systématique n'a guère été contributif du fait que le dépistage néonatal de la phénylcétonurie et de I'hypothyroïdie est généralisé à titre systématique par le test de Guthrie ${ }^{1}$ à la naissance en France.

\footnotetext{
${ }^{1}$ Le test de Guthrie, du nom de ce chercheur américain qui a mis au point la technique, permet le dépistage
}

Pour permettre une interprétation génotypique correcte, seuls les patients ayant subi une IRM cérébrale et une tomodensitométrie ont été inclus dans le dépistage ultérieur par séquençage. L’IRM cérébrale a détecté des anomalies manifestes, mais non spécifiques, isolées ou combinées dans $42 \%$ des cas [10] : il s'agissait d'hyper-intensités ponctuées de la substance blanche, d'anomalies de la différenciation substance grise/ blanche des cornes temporales et de dilatations des espaces de Wirchow-Robin (Tableau II).

Du fait des restrictions budgétaires, 141 patients seulement ont pu bénéficier d'un séquençage par exome ou sur panel. Une mutation monogénique causale a été reconnue responsable du TSA dans $23,4 \%$ des cas (33/141). La plupart des mutations sont survenues de novo (70\%), I'hérédité liée au chromosome $X$ rendant compte de $15 \%$ des cas et l'hétérozygotie composite de $15 \%$ des cas. Au total, 27 gènes de maladies différentes, responsables de TSA syndromique avec déficience intellectuelle, ont été identifiés dans cette étude (Tableau III).

Les consultations sur site ont ainsi permis de reconnaître des maladies génétiques méconnues chez 71 enfants atteints de TSA. Selon les parents, nommer la maladie n'a pas été perçu comme une «stigmatisation», mais plutôt comme un «soulagement», une «délivrance» qui les a aidés à comprendre et à surmonter l'épreuve, à établir des liens avec d'autres familles confrontées à des situations similaires. Occasionnellement, des couples se sont plaints que le conseil génétique était arrivé trop tard, alors qu'ils avaient déjà un enfant (ou apparenté) atteint, ou avaient abandonné le projet d'avoir un autre enfant. Pour les pédopsychiatres des établissements visités, nommer la maladie, stratifier la cohorte au plan étiologique ont été considérés comme des éléments positifs, leur permettant notamment d'accéder aux publications pertinentes et aux essais cliniques. $\varepsilon n$ l'absence de diagnostic d'organicité, des rendez-vous de suivi sur place ont été proposés aux familles et l'inclusion dans les programmes de recherche a été discutée (séquençage complet du génome et de l'exome).

\section{Discussion}

Vingt années de pratique nous ont appris que les consultations de génétique sur site dans des institutions spécialisées pour jeunes autistes contribuent à améliorer le standard de soins et à combattre la perte de

de cinq maladies génétiques rares potentiellement graves, dont des maladies métaboliques, la phénylcétonurie et l'hypothyroïdie, ainsi que l'hyperplasie congénitale des surrénales, la drépanocytose, et la mucoviscidose. 


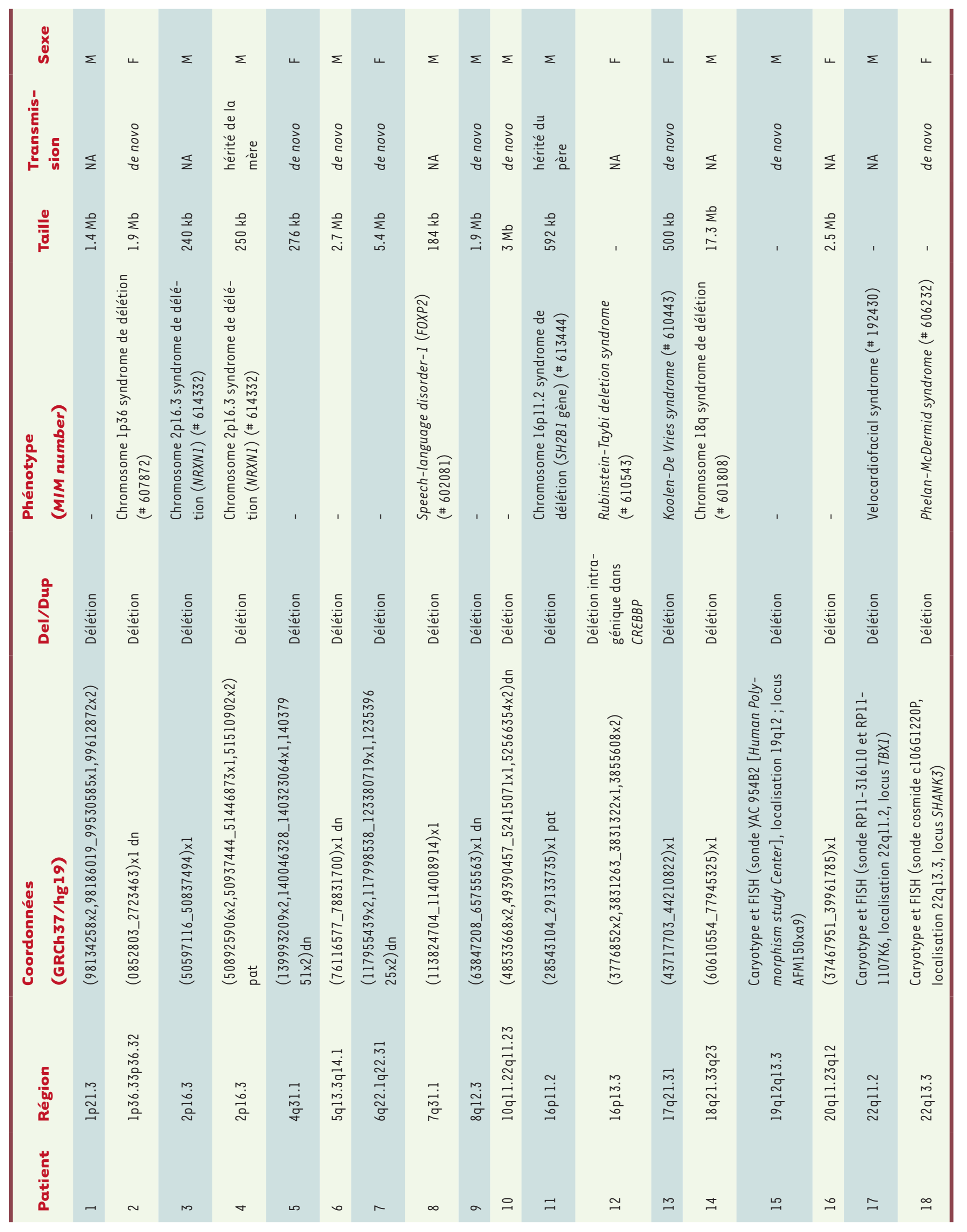



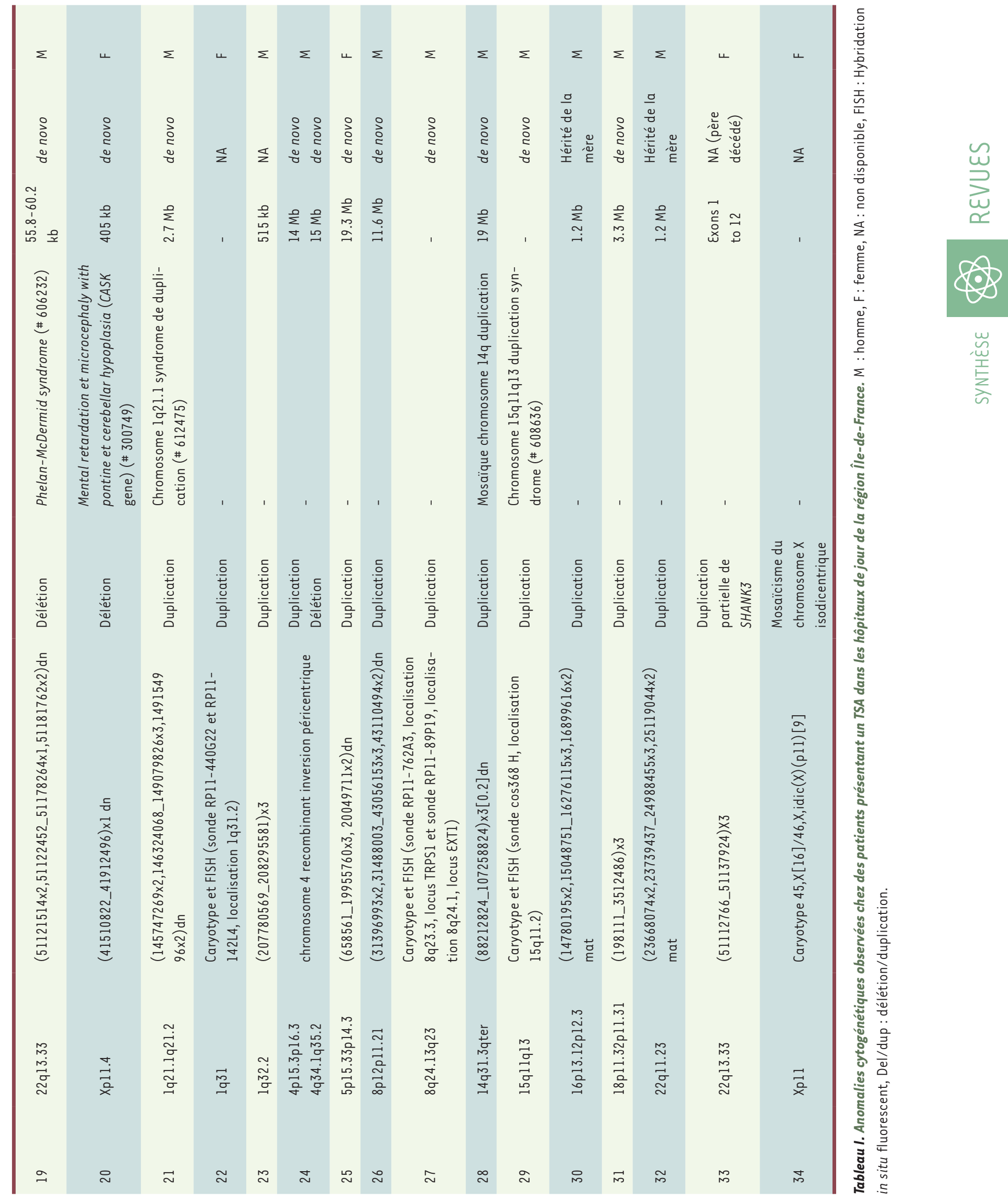


\begin{tabular}{|c|c|}
\hline Hyperintensité sous-corticale des pôles temporaux bilatéraux sur les images pondérées en T2 & $25 \%$ \\
\hline Hyperintensité de la substance blanche sur le T2 (hémisphères, périventriculaire, insula, pallidum, cervelet) & $18 \%$ \\
\hline Anomalie cérébelleuse (atrophie, hypoplasie, anomalie de signal) & $17 \%$ \\
\hline Corps calleux court, anormal ou épais & $13 \%$ \\
\hline Dilatation des espaces de Wirchow-Robin & $8 \%$ \\
\hline Anomalie de gyration (hétérorotopie, polymicrogyrie, pachygyrie) & $8 \%$ \\
\hline Kystes, tumeurs (tératomes, gangliomes, germinomes) & $10 \%$ \\
\hline Anomalies hypophysaires & $5 \%$ \\
\hline
\end{tabular}

Tableau II. Anomalies IRM observées chez 146 patients présentant un TSA dans les hôpitaux de jour de la région île-de-France.

chance diagnostique dans les TSA. Depuis 20 ans, une équipe mobile de génétique clinique se déplace dans 26 institutions spécialisées où 502 consultations ont été dispensées dans l'environnement familier des jeunes, réduisant ainsi l'angoisse de l'attente dans un lieu inhabituel. L'unité mobile de génétique médicale fonctionnant sous l'égide de I'hôpital Necker-Enfants Malades, les plates-formes des laboratoires hospitaliers et de l'Institut Imagine ont été accessibles, notamment pour la cytogénétique moléculaire, le séquençage à haut débit et I'IRM cérébrale. Grâce à cette organisation, un nombre considérable de consultations manquées ont pu être rattrapées, les enfants et leurs proches bénéficiant ainsi d'une consultation de génétique. Ce mode d'intervention nouveau a permis d'identifier une affection génétique préalablement méconnue chez 71 enfants atteints de TSA. Près de $10 \%$ des diagnostics concernaient des CNV pathogènes. Près d'un quart des patients testés en NGS $(23,4 \%)$ étaient porteurs de mutations dans 24 gènes différents, déjà connus pour être responsables de TSA $[5,6]$. Tous les patients diagnostiqués présentaient un TSA atypique et/ou syndromique avec une déficience intellectuelle modérée à sévère. Si on se souvient que les variants de signification inconnue ont été écartés et que les gènes rapportés dans une famille seulement n'ont pas été testés, ces résultats représentent certainement une estimation basse de la réalité. En revanche, aucun diagnostic génétique n'a été établi dans les cas d'autisme de haut niveau. Nous suggérons donc de proposer systématiquement une ACPA et un re-séquençage des gènes connus chez les enfants atteints de TSA syndromiques et/ou atypiques présentant une déficience intellectuelle associée. Bien que plus de 800 gènes responsables de TSA aient été rapportés [7], notre étude montre que les mutations de certains d'entre eux ont une incidence plus élevée dans les TSA syndromiques. En raison de l'accès limité aux plates-formes génomiques et de leur coût, une stratégie séquentielle consistant à tester en priorité un nombre réduit de gènes chez un nombre important d'enfants atteints de TSA syndromique pourrait être considérée.

Avant les consultations sur site, seule une faible fraction des patients avait été vue par un neuropédiatre ou un généticien. Si tant d'enfants ne sont pas explorés, c'est sans doute la conséquence du manque d'ex- perts, de l'insuffisance ou de l'inadaptation des consultations spécialisées et de la congestion des plateformes hospitalières, sous-dotées en biologistes qualifiés. De plus, si les parents acceptent d'être adressés à un neurologue pour leur enfant, la perception d'une consultation de génétique soulève davantage d'appréhensions. La possibilité de lancer des explorations génétiques est, par contre, bien mieux accueillie ultérieurement quand le diagnostic de TSA ne fait plus de doute, mais que de nombreuses questions concernant son mécanisme se posent aux parents. L'éponyme de TSA semble recouvrir un large éventail de situations, dont une fraction importante d'anomalies neuro-développementales. Eu égard aux difficultés rencontrées pour accéder à un établissement spécialisé, le fait d'avoir constaté des «caractéristiques autistiques » chez des enfants handicapés peut les avoir orientés vers ces institutions de qualité, indépendamment du diagnostic. Notre étude montre que les enfants admis dans ces institutions spécialisées, en région parisienne, ont un accès limité aux progrès de la génétique et que les consultations avancées sur site contribuent à remédier à cette situation pour les patients et les apparentés.

Bien que la découverte d'une affection génétique n'ait pas un impact immédiat sur la prise en charge, cette information est perçue par les parents comme un «soulagement », qui les a aidés à surmonter les difficultés et à dépasser le sentiment de culpabilité d'avoir donné naissance à un enfant atteint de TSA. Les associations de parents confrontés à des situations similaires jouent un rôle important, d'autant que la constitution de cohortes relativement homogènes est un pré-requis pour les études relatives à l'histoire naturelle des soustypes de TSA.

Les consultations sur site permettent en outre de formuler des préconisations personnalisées [11, 12]. Des 


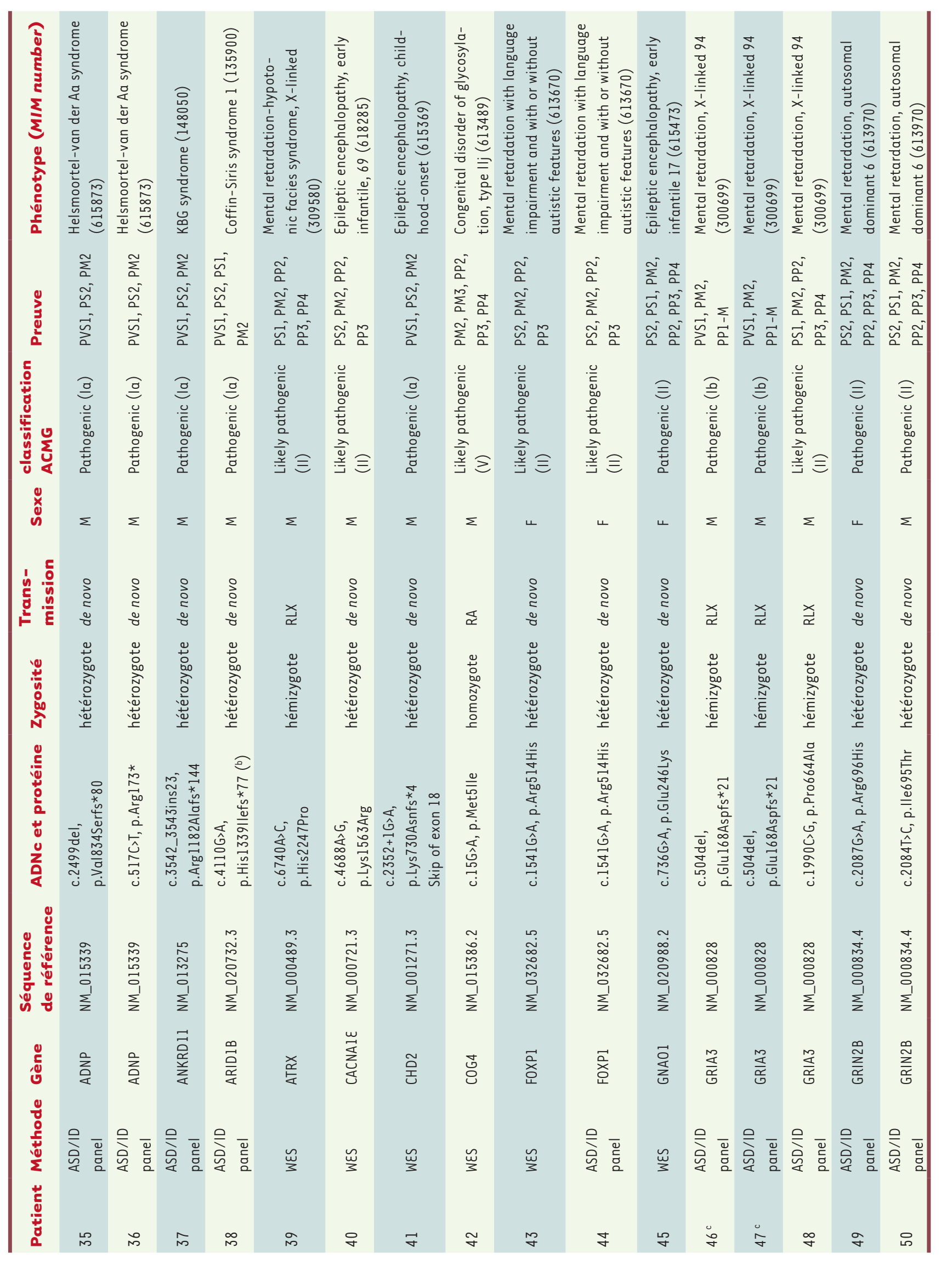

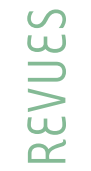

$\notin$ 


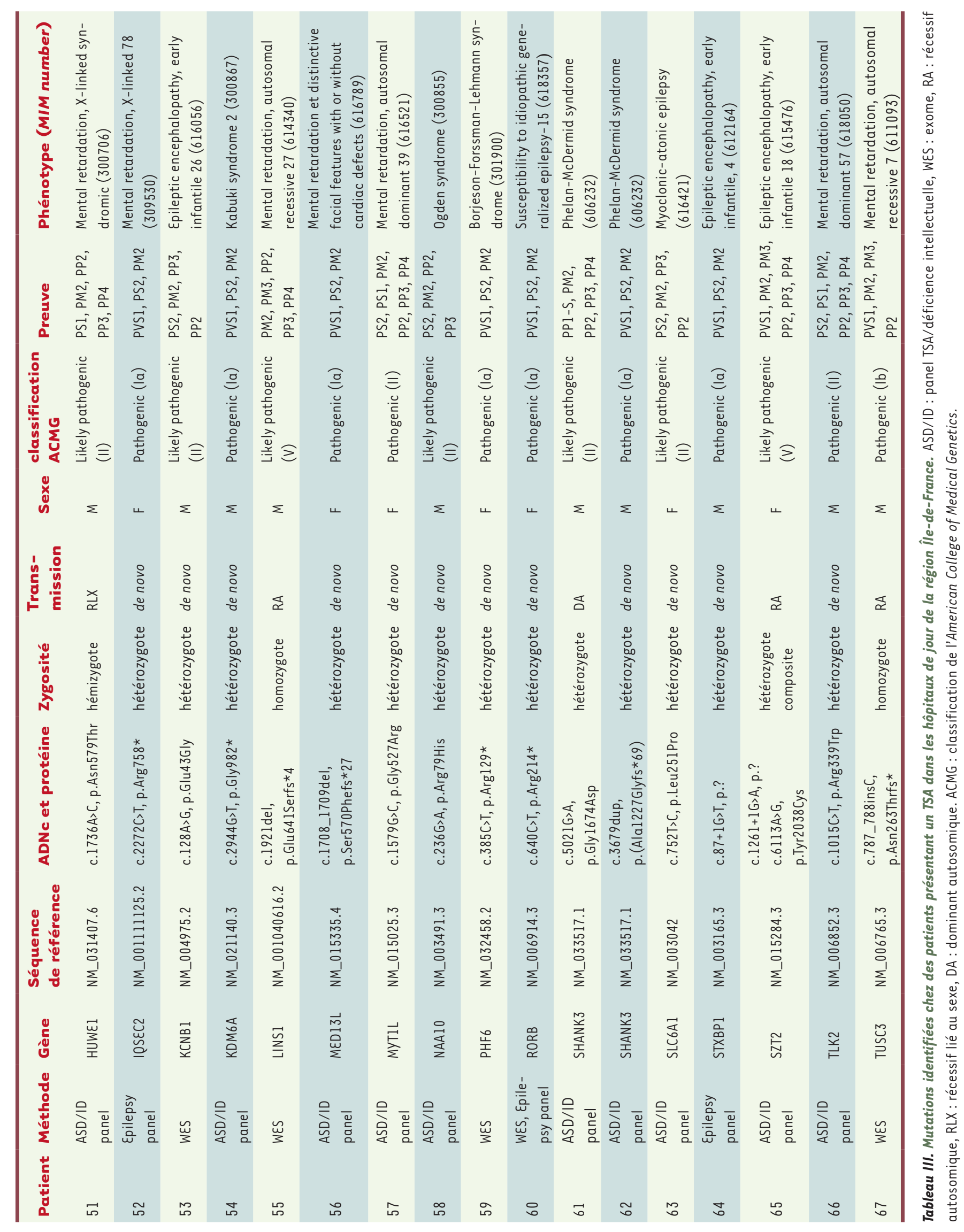


évaluations neuropsychologiques ont pu être proposées aux patients diagnostiqués afin de mieux caractériser ces formes génétiques de TSA, de développer de nouvelles stratégies de remédiation cognitive et de proposer des prises en charge cognitivo-comportementales personnalisées. Les consultations sur site ont également eu un impact immédiat sur le conseil génétique, en particulier lorsque des mutations de novo et des CNV ont été identifiés car ils excluent a priori le risque de récurrence pour les parents et les apparentés. Certains couples se sont plaints que ce conseil arrivait trop tard alors qu'ils avaient déjà un deuxième enfant atteint (ou un apparenté) ou avaient abandonné le projet d'avoir un autre enfant. Omettre ou différer les consultations de génétique médicale et ne pas mettre en garde contre un éventuel risque génétique peut avoir de graves conséquences sur les formes héréditaires de TSA.

La sous-utilisation des services génétiques par les familles concernées n'est pas spécifique à la France et représente un défi majeur [13]. Une étude réalisée en Espagne a exploré l'accès aux services génétiques et la perception du risque génétique chez les parents d'enfants autistes [14]. Elle a révélé une sous-utilisation frappante des services, avec seulement $30 \%$ des familles ayant consulté et $13 \%$ des patients ayant fait le test génétique recommandé. De même, une récente étude taïwanaise a révélé que $2 / 3$ des parents d'enfants atteints de TSA n'avaient jamais entendu parler de tests génétiques pour les TSA, bien que la majorité d'entre eux $(71,4 \%)$ exprime un intérêt pour ces tests [15].

\section{En conclusion}

Notre étude montre que les enfants atteints de TSA admis dans les établissements spécialisés de la région parisienne ont un accès limité au progrès médical et aux explorations qui pourraient leur être proposées. Nous suggérons de considérer les consultations avancées sur site comme une solution permettant d'améliorer la qualité des soins et la diffusion du progrès médical pour les personnes concernées et leurs apparentés. $\diamond$

\section{SUMMARY}

Twenty years of on-site clinical genetics consultations for people with ASD

Despite advances in neurogenetics of autism spectrum disorders (ASD), many patients fail to be systematically investigated, owing to preconceived ideas, limited access to genetics facilities and inadequacy of consultations to children with behavioural problems. To improve access to services, we reversed the paradigm and delivered on-site genetics consultations to ASD children of Greater Paris day care hospitals and specialized institutions. Since 1998, an ambulatory medical genetics team has been in operation, offering on-site consultations and services to patients and relatives in their usual environment. Because the mobile medical genetics unit operates under the umbrella of a university hospital, service laboratories were shared, including molecular cytogenetics and next generation sequencing (NGS). For the past 20 years, 502 patients from 26 institutions benefited from on-site consultations and genetics services in their usual environment. Less than $1 \%$ of parents declined the offer. Previously undiagnosed genetics conditions were recognized in 71 ASD children, including pathogenic CNV variants (34/388: 8.8; de novo: 19, inherited : 4), Fragile X (4/312: $1.3 \%)$ and deleterious variants in disease causing genes $(33 / 141$; $23.4 \%$ : de novo : 23 ; inherited: 10 , including 5 X-linked and 5 compound heterozygote mutations). Brain MRI were possible in 347 patients and $42 \%$ were considered abnormal (146/347). All diagnosed patients presented atypical/syndromic ASD with moderate to severe intellectual disability. Thanks to such flexible organisation, a considerable number of missed consultations were tracked and families first benefited from medical genetics services. Owing to constraints imposed by behavioural problems in ASD, we suggest considering on-site genetics services to implement standard of care and counteract the loss of chance to patients and relatives. $\diamond$

\section{LIENS D'INTÉRÊT}

Les auteurs déclarent n'avoir aucun lien d'intérêt concernant les données publiées dans cet article.

\section{RÉFÉRENCES}

1. Charman T. The new genetics of autism : a translational opportunity? Lancet Psychiatry 2015; $10: 856-7$.

2. Lai MC, Lombardo MV, Baron-Cohen S. Autism. Lancet 2014 ; 383 : 896-910.

3. DSM-V. Diagnostic and statistical manual of mental disorders, 5 th ed. Washington DC : American Psychiatric Association, 2013.

4. Leroy C, Jacquemont ML, Doray B. Xq25 duplication : the crucial role of the STAG2 gene in this novel human cohesinopathy. Clin Genet $2016 ; 89: 68-73$

5. Redin C, Gerard B, Lauer J. Efficient strategy for the molecular diagnosis of intellectual disability using targeted high-throughput sequencing. J Med Genet $2014 ; 51: 724-36$.

6. Yin J, Schaaf CP. Autism genetics - an overview. Prenat Diagn 2017 ; 37 : $14-30$

7. Morgan A, Gandin I, Belcaro C. Target sequencing approach intended to discover new mutations in non-syndromic intellectual disability. Mutat Res $2015 ; 781: 32-6$.

8. Grozeva D, Carss K, Spasic-Boskovic 0. Targeted next-generation sequencing analysis of 1,000 individuals with intellectual disability. Hum Mutat 2015 ; $36: 1197-204$.

9. Martínez F, Caro-Llopis A, Roselló M. High diagnostic yield of syndromic intellectual disability by targeted next-generation sequencing. J Med Genet $2017 ; 54: 87-92$.

10. Boddaert N, Zilbovicius M, Philippe A. MRI findings in 77 children with nonsyndromic autistic disorders. PLoS One $2009 ; 4$ : e4415.

11. Vorstman JAS, Parr JR, Moreno-De-Luca D, Anney RJL. Autism genetics : opportunities and challenges for clinical translation. Nat Rev Genet 2017 ; $18: 362-76$.

12. Poisson $A$, Nicolas $A$, Cochat $P$. Behavioral disturbance and treatment strategies in Smith-Magenis syndrome. Orphanet J Rare Dis $2015 ; 10: 111$.

13. Lord C, Elsabbagh M, Baird G, Veenstra-Vanderweele J. Autism spectrum disorder. Lancet $2018 ; 392:$ 508-20.

14. Codina-Solà M, Pérez-Jurado LA, Cusco I, Serra-Juhé C. Provision of genetic services for autism and its impact on Spanish families. J Autism Dev Disord $2017 ; 47: 2947-56$.

15. Chen LS, Min J, Zhao S, et al. Information needs in genetic testing : A needs assessment survey among Taiwanese parents of children with autism spectrum disorders. Autism 2018 ; Aug 3 : 1362361318778903 . doi : $10.1177 / 1362361318778903$.

16. Munnich A, Demily C, Frugère L, et al. Impact of on-site clinical genetics consultations on diagnostic rate in children and young adults with autism spectrum disorder. Molecular Autism 2019; 10 : 33. https://molecularautism. biomedcentral.com/track/pdf/10.1186/s13229-019-0284-2 